\title{
Robust stabilization with positive real uncertainty: Beyond the small gain theorem *
}

\author{
Wassim M. Haddad \\ Department of Mechanical and Aerospace Engineering. Florida Institute of Technology, Melbourne, FL 32901, USA
}

\author{
Dennis S. Bernstein \\ Harris Corporation, Government Aerospace Systems Division, MS 22/4847, Melbourne, FL 32902, USA
}

Received 17 September 1990

Revised 15 March 1991

Abstract: In many applications of feedback control, phase information is available concerning the plant uncertainty. For example, lightly damped flexible structures with colocated rate sensors and force actuators give rise to positive real transfer functions. Closed-loop stability is thus guaranteed by means of negative feedback with strictly positive real compensators. In this paper, the properties of positive real transfer functions are used to guarantee robust stability in the presence of positive real (but otherwise unknown) plant uncertainty. These results are then used for controller synthesis to address the problem of robust stabilization in the presence of positive real uncertainty. One of the principal motivations for these results is to utilize phase information in guaranteeing robust stability. In this sense these results go beyond the usual limitations of the small gain theorem and quadratic Lyapunov functions which may be conservative when phase information is available. The results of this paper are based upon a Riccati equation formulation of the positive real lemma and thus are in the spirit of recent Riccati-based approaches to bounded real $\left(\mathrm{H}_{\infty}\right)$ control.

Keywords: Bounded real; positive real; robust stabilization; phase information.

\section{Introduction}

In many applications of feedback control, phase information is available concerning the plant uncertainty. For example, lightly damped flexible structures with colocated rate sensors and force actuators give rise to positive real transfer functions. Closed-loop stability is thus guaranteed by means of negative feedback with strictly positive real compensators. This principle has been widely used to design robust controllers for flexible structures $[8,10,16,25,29,30,31,34,37,38]$.

The salient feature of positive real transfer functions is that they are dissipative and phase bounded $[1-7,19,20,26,33,39,40,46,47,50,51]$. Hence the feedback interconnection of positive real transfer functions is guaranteed to be stable without requiring that a small gain condition [53] be satisfied. Positive real design is thus potentially less conservative than bounded real $\left(\mathrm{H}_{\infty}\right)$ design in the presence of phase information.

In this paper we utilize properties of positive real transfer functions to develop new conditions for robust stability and robust stabilizability. Although related results have been developed previously $[9,17,35,44,45]$, this paper goes beyond earlier work by exploiting a Riccati equation formulation in the spirit of recent advances in $H_{\infty}$ synthesis [14,21,24,32,42,54]. This is done in two different, but equivalent, ways. First we show that the Riccati equation used to enforce an $\mathrm{H}_{\infty}$ constraint can be transformed to yield a different Riccati equation that enforces a positive real constraint (Theorem 3.2). Alternatively, we show that the same Riccati equation can be obtained by manipulating the conditions of the positive real

* This research was supported in part by the Air Force Office of Scientific Research under contracts F49620-89-C-0011 and F49620-89-C-0029. 
lemma (Proposition 3.3). Many of the techniques and transformations used in these steps are due to [6], which contains an extensive treatment of positive real and bounded real transfer functions.

Once the Riccati equation that enforces positive realness has been derived, robust stability can be guaranteed for a class of perturbations involving an arbitrary constant positive real matrix (see the set $\mathscr{U}$ defined by (4.5) and Theorem 4.1). The modeling of matrix uncertainty by means of a "fictitious" feedback loop (linear fractional transformation) is directly analogous to the small gain $\left(\mathrm{H}_{\infty}\right)$ parameter uncertainty model of [32]. In our case, however, the class of uncertainties includes a phase constraint rather than a small gain condition.

Having enforced robust stability for positive real uncertainty, we then proceed in Section 5 to give sufficient conditions for robust stabilizability in terms of a pair of coupled algebraic Riccati equations (Theorem 5.1). A robustly stabilizing feedback gain is then given in terms of the solutions to the Riccati equations. The stabilizability result is first stated for static output feedback and then specialized to the case of full-state feedback. Necessary conditions for robust stabilization with positive real uncertainty will be considered in a future paper.

Finally, we close the paper by discussing connections between the positive real uncertainty modeling approach of this paper and the Maximum Entropy approach to robust control design of [11-13,16,18,27,28].

\section{Notation}

$\mathbb{R}, \mathbb{R}^{r \times s}$

$I_{r}, I ;()^{\mathrm{T}},()^{*}$

tr, $\rho(), \sigma_{\max }()$

$\|H(s)\|_{\infty}$

$n, m, m_{0}, l$

$A, B, C, K$

$B_{0}, C_{0}, D_{0}, F$ real numbers, $r \times s$ real matrices.

$r \times r$ identity matrix; transpose, complex conjugate transpose, trace, spectral radius, largest singular value.

$\sup _{\omega \in \mathbf{R}} \sigma_{\max }[H(\mathrm{j} \omega)]$.

positive integers.

$n \times n, n \times m, l \times n, m \times l$ matrices.

$n \times m_{0}, m_{0} \times n, m_{0} \times m_{0}, m_{0} \times m_{0}$ matrices.

\section{Preliminaries}

In this section we establish key definitions and notational conventions that simplify the exposition in later sections. We begin with the definitions of positive real and bounded real transfer functions $[1,6]$.

In this paper a real-rational matrix function is a matrix whose elements are rational functions with real coefficients. Furthermore, a transfer function is a real-rational matrix function each of whose elements is proper, i.e., finite at $s=\infty$. Finally, a stable transfer function is a transfer function each of whose poles is in the open left half plane. The space of stable transfer functions is denoted in [22] by $\mathrm{RH}_{\infty}$, i.e., the real-rational subset of $\mathrm{H}_{\infty}$.

A square transfer function $G(s)$ is called positive real $[6$, p. 216] if (1) all elements of $G(s)$ are analytic for $\operatorname{Re}[s]>0$ and (2) $G(s)+G^{*}(s)$ is nonnegative-definite for $\operatorname{Re}[s]>0$. A square transfer function $G(s)$ is called strictly positive real $[10,36,48]$ if (1) all elements of $G(s)$ are analytic for $\operatorname{Re}[s] \geq 0$ and (2) $G(\mathrm{j} \omega)+G^{*}(\mathrm{j} \omega)$ is positive definite for all real $\omega$. Finally, a square transfer function $G(s)$ is strongly positive real if it is strictly positive real and $D+D^{\mathrm{T}}>0$, where $D:=G(\infty)$. Note that strongly positive real implies strictly positive real, which further implies positive real. Furthermore, we note that if a transfer function is strictly positive real, then the system is stable and dissipative.

Next, we give the definition of bounded real. A transfer function $H(s)$ is bounded real [6] if and only if (1) all elements of $H(s)$ are analytic for $\operatorname{Re}[s] \geq 0$ and (2) $I-H(\mathrm{j} \omega) H^{*}(\mathrm{j} \omega)$ is nonnegative definite for all real $\omega$. Equivalently, (2) can be replaced by [6, p. 307] $\left(2^{\prime}\right) I-H(s) H^{*}(s)$ is nonnegative definite for $\operatorname{Re}[s]>0$. Alternatively, a transfer function $H(s)$ is bounded real if and only if $H(s)$ is stable and satisfies $\|H(s)\|_{\infty} \leq 1$. 
Next we establish some notation involving state space realizations of transfer functions. Let [22]

$$
G(s) \sim\left[\begin{array}{c|c}
A & B \\
\hline C & D
\end{array}\right]
$$

denote a state space realization of $G(s)$, that is, $G(s)=C(s I-A)^{-1} B+D$. If $G(s)$ is square and det $D \neq 0$, then

$$
G^{-1}(s) \sim\left[\begin{array}{c|c}
A-B D^{-1} C & B D^{-1} \\
\hline-D^{-1} C & D^{-1}
\end{array}\right] .
$$

Finally, if $G_{1}(s)=C_{1}\left(s I-A_{1}\right)^{-1} B_{1}+D_{1}$ and $G_{2}(s)=C_{2}\left(s I-A_{2}\right)^{-1} B_{2}+D_{2}$, then

$$
G_{1}(s) G_{2}(s) \sim\left[\begin{array}{cc|c}
A_{2} & 0 & B_{2} \\
B_{1} C_{1} & A_{1} & B_{1} D_{2} \\
\hline D_{1} C_{2} & C_{1} & D_{1} D_{2}
\end{array}\right] .
$$

\section{Riccati equation characterizations of positive real and bounded real transfer functions}

In this section we provide explicit connections between positive real and bounded real transfer functions and their associated state-space realizations. Furthermore, we give Riccati equation characterizations of their resulting state-space realizations. Finally, we draw connections with the well-known positive real lemma $[1,6,20]$.

We begin with a result [6] that relates bounded real transfer functions to positive real transfer functions via the Cayley (bilinear) transform. Throughout the paper $\gamma$ denotes a positive number.

Lemma 3.1. If $\gamma^{-1} H(s)$ is an $m \times m$ bounded real transfer function with $\operatorname{det}\left[I_{m}-\gamma^{-1} H(s)\right] \neq 0$ for $\operatorname{Re}[s]>0$, then

$$
G(s):=\left[I_{m}-\gamma^{-1} H(s)\right]^{-1}\left[I_{m}+\gamma^{-1} H(s)\right]
$$

is positive real. Conversely, if $G(s)$ is an $m \times m$ positive real transfer function such that $G(s)$ is analytic for $\operatorname{Re}[s] \geq 0$, then

$$
\gamma^{-1} H(s):=\left[G(s)-I_{m}\right]\left[G(s)+I_{m}\right]^{-1}
$$

is bounded real.

Proof. Suppose $\gamma^{-1} H(s)$ is bounded real. Since $\operatorname{det}\left[I_{m}-\gamma^{-1} H(s)\right] \neq 0$ for $\operatorname{Re}[s]>0$, it follows that $G(s)$ is analytic for $\operatorname{Re}[s]>0$. Then with $G(s)$ defined by (3.1) it follows that $\gamma^{-1} H(s)$ satisfies (3.2). Thus, we obtain for $\operatorname{Re}[s]>0$,

$$
\gamma^{-2} H(s) H^{*}(s)=\left[G(s)-I_{m}\right]\left[G(s)+I_{m}\right]^{-1}\left[G^{*}(s)+I_{m}\right]^{-1}\left[G^{*}(s)-I_{m}\right] \leq I_{m},
$$

which implies

$$
\left[G(s)+I_{m}\right]^{-1}\left[G^{*}(s)+I_{m}\right]^{-1} \leq\left[G(s)-I_{m}\right]^{-1}\left[G^{*}(s)-I_{m}\right]^{-1}
$$

or, equivalently,

$$
\left[G^{*}(s)+I_{m}\right]\left[G(s)+I_{m}\right] \geq\left[G^{*}(s)-I_{m}\right]\left[G(s)-I_{m}\right]
$$


which further implies that $G(s)+G^{*}(s) \geq 0$ is $\operatorname{Re}[s]>0$. Conversely, suppose $G(s)$ is positive real. Then, since $G(s)$ is assumed to be analytic for $\operatorname{Re}[s] \geq 0$, it is easy to show that $\operatorname{det}\left[G(s)+I_{m}\right] \neq 0$ for $\operatorname{Re}[s] \geq 0$. Therefore, $\gamma^{-1} H(s)$ defined by (3.2) is analytic for $\operatorname{Re}[s] \geq 0$. Then with $\gamma^{-1} H(s)$ defined by (3.2) it follows that $G(s)$ satisfies (3.1). Next, for $\operatorname{Re}[s]>0$ we obtain

$$
\begin{aligned}
G(s)+G^{*}(s) & =\left[I_{m}-\gamma^{-1} H(s)\right]^{-1}\left[I_{m}+\gamma^{-1} H(s)\right]+\left[I_{m}+\gamma^{-1} H^{*}(s)\right]\left[I_{m}-\gamma^{-1} H^{*}(s)\right]^{-1} \\
& \geq 0
\end{aligned}
$$

Forming $\left[I_{m}-\gamma^{-1} H(s)\right](3.6)\left[I_{m}-\gamma^{-1} H^{*}(s)\right]$ yields

$$
\left[I_{m}+\gamma^{-1} H(s)\right]\left[I_{m}-\gamma^{-1} H^{*}(s)\right]+\left[I_{m}-\gamma^{-1} H(s)\right]\left[I_{m}+\gamma^{-1} H^{*}(s)\right] \geq 0,
$$

which implies $I_{m}-\gamma^{-2} H(s) H^{*}(s) \geq 0$ for $\operatorname{Re}[s]>0$.

Next, we use the results of Lemma 3.1 to establish connections between the state space realizations of positive real and bounded real transfer functions. First, however, we give a key lemma concerning positive real and strictly positive real matrices.

Lemma 3.2. Let $M, N \in \mathbb{C}^{n \times n}$ be such that $M+M^{*} \geq 0$ and $N+N^{*}>0$. Then $\operatorname{det}\left(\alpha I_{n}+M N\right) \neq 0$ for all $\alpha>0$.

Proof. First we show that $N$ is invertible. Let $x \in \mathbb{C}^{n}, x \neq 0$, and $\lambda \in \mathbb{C}$ be such that $N x=\lambda x$ and hence $x^{*} N^{*}=\bar{\lambda} x^{*}$. Then $x^{*}\left(N+N^{*}\right) x>0$ implies that $\operatorname{Re} \lambda>0$. Hence det $N \neq 0$. Now let $\alpha>0$ and define $S:=\alpha N^{-1}+M$. Now, since $N^{-1}+N^{-*}=N^{-1}\left(N+N^{*}\right) N^{-*}>0$, it follows that $S+S^{*}>0$. Thus $\operatorname{det} S \neq 0$. Consequently,

$$
\operatorname{det}\left(\alpha I_{n}+M N\right)=\operatorname{det} N S=(\operatorname{det} N)(\operatorname{det} S) \neq 0 .
$$

Proposition 3.1. If $G(s)$ is a positive real transfer function with minimal realization

$$
G(s) \sim\left[\begin{array}{c|c}
A & B \\
\hline C & D
\end{array}\right]
$$

then the bounded real transfer function $\gamma^{-1} H(s)$ defined by (3.2) has a minimal realization

$$
\gamma^{-1} H(s) \sim\left[\begin{array}{c|c}
\hat{A} & \hat{B} \\
\hline \hat{C} & \hat{D}
\end{array}\right],
$$

where

$$
\begin{aligned}
& \hat{A}:=A-B\left(I_{m}+D\right)^{-1} C, \quad \hat{B}:=\sqrt{2} B\left(I_{m}+D\right)^{-1}, \\
& \hat{C}:=\sqrt{2}\left(I_{m}+D\right)^{-1} C, \quad \hat{D}:=\left(D-I_{m}\right)\left(D+I_{m}\right)^{-1} .
\end{aligned}
$$

Conversely, if $\gamma^{-1} H(s)$ is an $m \times m$ bounded real transfer function such that $\operatorname{det}\left[I_{m}-\gamma^{-1} H(s)\right] \neq 0$ for $\operatorname{Re}[s]>0$ and with minimal realization

$$
\gamma^{-1} H(s) \sim\left[\begin{array}{l|l}
A & B \\
\hline C & D
\end{array}\right]
$$

then the positive real transfer function $G(s)$ defined by (3.1) has a minimal realization

$$
G(s) \sim\left[\begin{array}{c|c}
\bar{A} & \bar{B} \\
\hline \bar{C} & \bar{D}
\end{array}\right],
$$


where

$$
\begin{aligned}
& \bar{A}:=A+B\left(I_{m}-D\right)^{-1} C, \quad \bar{B}:=\sqrt{2} B\left(I_{m}-D\right)^{-1}, \\
& \bar{C}:=\sqrt{2}\left(I_{m}-D\right)^{-1} C, \quad \bar{D}:=\left(I_{m}-D\right)^{-1}\left(I_{m}+D\right) .
\end{aligned}
$$

Proof. Given (3.7) it follows that the realizations of $G(s)-I_{m}$ and $G(s)+I_{m}$ are given by

$$
\left[G(s)-I_{m}\right] \sim\left[\begin{array}{c|c}
A & B \\
\hline C & D-I_{m}
\end{array}\right], \quad\left[G(s)+I_{m}\right] \sim\left[\begin{array}{c|c}
A & B \\
\hline C & D+I_{m}
\end{array}\right] .
$$

Now, since $G(s)$ is positive real, it follows that $D+D^{\mathrm{T}} \geq 0$ which further implies by Lemma 3.2 that $I_{m}+D$ is invertible. Next, using (2.2) we have

$$
\left[G(s)+I_{m}\right]^{-1} \sim\left[\begin{array}{c|c}
A-B\left(I_{m}+D\right)^{-1} C & B\left(I_{m}+D\right)^{-1} \\
\hline-\left(I_{m}+D\right)^{-1} C & \left(D+I_{m}\right)^{-1}
\end{array}\right]
$$

Using (2.3), it now follows that $\gamma^{-1} H(s)=\left[G(s)-I_{m}\right]\left[G(s)+I_{m}\right]^{-1}$ has a nonminimal realization

$$
\gamma^{-1} H(s) \sim\left[\begin{array}{cc|c}
A-B\left(I_{m}+D\right)^{-1} C & 0 & B\left(I_{m}+D\right)^{-1} \\
-B\left(I_{m}+D\right)^{-1} C & A & B\left(I_{m}+D\right)^{-1} \\
\hline\left(I_{m}-D\right)\left(I_{m}+D\right)^{-1} C & C & \left(D-I_{m}\right)\left(D+I_{m}\right)^{-1}
\end{array}\right] .
$$

Next it follows from state-space manipulations that $\gamma^{-1} H(s)$ has a minimal state-space realization given by

$$
\gamma^{-1} H(s) \sim\left[\begin{array}{c|c}
A-B\left(I_{m}+D\right)^{-1} C & \sqrt{2} B\left(I_{m}+D\right)^{-1} \\
\hline \sqrt{2}\left(I_{m}+D\right)^{-1} C & \left(D-I_{m}\right)\left(D+I_{m}\right)^{-1}
\end{array}\right] .
$$

Furthermore, Lemma 3.1 implies that $\gamma^{-1} H(s)$ is bounded real. Finally, the converse is shown in a similar fashion.

Having established connections between state-space realizations of positive real and bounded real transfer functions we proceed in the spirit of recent $H_{\infty}$ results $[14,21,24,32,42,54]$ to establish Riccati equation characterizations of positive real systems.

Theorem 3.1. Let

$$
H(s) \sim\left[\begin{array}{c|c}
A & B \\
\hline C & D
\end{array}\right], \quad \text { where } \sigma_{\max }(D)<\gamma .
$$

If there exists an $n \times n$ nonnegative-definite matrix $Q$ satisfying

$$
0=A Q+Q A^{\mathrm{T}}+\gamma^{-2}\left(B D^{\mathrm{T}}+Q C^{\mathrm{T}}\right)\left(I_{m}-\gamma^{-2} D D^{\mathrm{T}}\right)^{-1}\left(B D^{\mathrm{T}}+Q C^{\mathrm{T}}\right)^{\mathrm{T}}+B B^{\mathrm{T}},
$$

then $(A, B)$ is stabilizable if and only if

$A$ is asymptotically stable.

Furthermore, in this case,

$$
\|H(s)\|_{\infty} \leq \gamma .
$$


Conversely, if $A$ is asymptotically stable and $\|H(s)\|_{\infty}<\gamma$, then there exists a unique nonnegative-definite matrix $Q$ satisfying (3.18) and such that the eigenvalues of

$$
A+\gamma^{-2} B D^{\mathrm{T}}\left(I_{m}-\gamma^{-2} D D^{\mathrm{T}}\right)^{-1} C+\gamma^{-2} Q C^{\mathrm{T}}\left(I_{m}-\gamma^{-2} D D^{\mathrm{T}}\right)^{-1} C
$$

lie in the open left half plane. Furthermore, $Q$ is the minimal solution to (3.18).

Proof. The asymptotic stability of $A$ follows directly from Lyapunov theory while (3.20) follows from algebraic manipulation of (3.18); for details see [24]. The converse follows from the bounded real lemma [6, p. 308] or from spectral factor theory [49]. Finally, the proof of minimality is given in [52].

Next, we utilize a transformation that converts a nonstrictly proper transfer function into a strictly proper transfer function both of which satisfy the same $\mathrm{H}_{\infty}$ bound. For convenience in stating this result define the notation

$$
M:=I_{m}-\gamma^{-2} D D^{\mathrm{T}}, \quad N:=I_{m}-\gamma^{-2} D^{\mathrm{T}} D .
$$

Note that $M$ is positive definite if and only if $N$ is positive definite.

Proposition 3.2. Let

$$
H(s) \sim\left[\begin{array}{c|c}
A & B \\
\hline C & D
\end{array}\right]
$$

Then $A$ is asymptotically stable, $M>0$, and

$$
\|H(s)\|_{\infty}<\gamma
$$

if and only if $A^{\prime}$ is asymptotically stable and

$$
\left\|H^{\prime}(s)\right\|_{\infty}<\gamma, \quad \text { where } H^{\prime}(s) \sim\left[\begin{array}{c|c}
A^{\prime} & B^{\prime} \\
\hline C^{\prime} & 0
\end{array}\right]
$$

with

$$
A^{\prime}:=A+\gamma^{-2} B D^{\mathrm{T}} M^{-1} C, \quad B^{\prime}:=B N^{-1 / 2}, \quad C^{\prime}:=M^{-1 / 2} C .
$$

Furthermore, (3.18) is equivalent to

$$
0=A^{\prime} Q+Q A^{\prime \mathrm{T}}+\gamma^{-2} Q C^{\prime \top \mathrm{T}} C^{\prime} Q+B^{\prime} B^{\prime \mathrm{T}}
$$

Proof. Note that $\|H(s)\|_{\infty}<\gamma$ implies that $M>0$. The results now follow from Theorem 3.1 and algebraic manipulation. For details see [24].

Next, using Theorem 3.1 we give a Riccati equation characterization of positive real transfer functions. To do this we use (3.18) to imply that the transfer function corresponding to $(\hat{A}, \gamma \hat{B}, \hat{C}, \gamma \hat{D})$ has $\mathrm{H}_{\infty}$ norm less than $\gamma$. By Lemma 3.1 and Proposition 3.1 the resulting Riccati equation, i.e., (3.18) with $(A, B, C, D)$ replaced by $(\hat{A}, \gamma \hat{B}, \hat{C}, \gamma \hat{D})$, implies that

$$
G(s) \sim\left[\begin{array}{c|c}
A & B \\
\hline C & D
\end{array}\right]
$$

is positive real. To utilize Theorem 3.1 we require that $\sigma_{\max }(\gamma \hat{D})<\gamma$ or, equivalently,

$$
I_{m}-\hat{D} \hat{D}^{\mathrm{T}}>0
$$


Now, using (3.12), condition (3.27) is equivalent to

$$
I_{m}-\left(D-I_{m}\right)\left(D+I_{m}\right)^{-1}\left(D+I_{m}\right)^{-\mathrm{T}}\left(D-I_{m}\right)^{\mathrm{T}}>0 .
$$

Since $D+I_{m}$ and $D-I_{m}$ commute, (3.28) implies

$$
\left(I_{m}-D\right)\left(I_{m}-D^{\mathrm{T}}\right)<\left(I_{m}+D\right)\left(I+D^{\mathrm{T}}\right),
$$

which implies that

$$
D+D^{\mathrm{T}}>0 \text {. }
$$

Thus, we restrict our attention to strongly positive real systems.

Theorem 3.2. Let

$$
G(s) \sim\left[\begin{array}{c|c}
A & B \\
\hline C & D
\end{array}\right],
$$

define $\hat{A}, \hat{B}, \hat{C}, \hat{D}$ by (3.9)-(3.12), and assume $\sigma_{\max }(\hat{D})<1$. If there exists an $n \times n$ nonnegative-definite matrix 2 satisfying

$$
0=\hat{A} \mathscr{Q}+\mathscr{2} \hat{A}^{\mathrm{T}}+\left(\hat{B} \hat{D}^{\mathrm{T}}+\mathscr{2} \hat{C}^{\mathrm{T}}\right)\left(I_{m}-\hat{D} \hat{D}^{\mathrm{T}}\right)^{-1}\left(\hat{B} \hat{D}^{\mathrm{T}}+\mathscr{2} \hat{C}^{\mathrm{T}}\right)^{\mathrm{T}}+\hat{B} \hat{B}^{\mathrm{T}}
$$

$(\hat{A}, \hat{B})$ is stabilizable, and

$$
\operatorname{det}\left[I_{m}-\hat{C}\left(s I_{n}-\hat{A}\right)^{-1} \hat{B}-\hat{D}\right] \neq 0 \quad \text { for } \operatorname{Re}[s]>0,
$$

then

$$
G(s) \text { is positive real. }
$$

Conversely, if $\hat{A}$ is asymptotically stable and $G(s)$ is strongly positive real, then there exists $a$ unique nonnegative-definite matrix $\mathcal{Q}$ satisfying (3.31).

Proof. The result is a direct consequence of Theorem 3.1, Proposition 3.1 and Lemma 3.1.

Remark 3.1. Using Proposition 3.2 we can represent (3.31) in the equivalent form

$$
0=\hat{A}^{\prime} \mathscr{Q}+\mathscr{Q} \hat{A}^{\prime \mathrm{T}}+\mathscr{2} \hat{C}^{\prime \mathrm{T}} \hat{C}^{\prime} \mathscr{Q}+\hat{B}^{\prime} \hat{B}^{\prime \mathrm{T}},
$$

where

$$
\begin{aligned}
& \hat{A}^{\prime}:=A-B\left(I_{m}+D\right)^{-1} C+2 B\left(I_{m}+D\right)^{-1} \hat{D}^{\mathrm{T}}\left(I_{m}-\hat{D} \hat{D}^{\mathrm{T}}\right)^{-1}\left(I_{m}+D\right)^{-1} C, \\
& \hat{B}^{\prime}:=\sqrt{2} B\left(I_{m}+D\right)^{-1}\left(I_{m}-\hat{D}^{\mathrm{T}} \hat{D}\right)^{-1 / 2} \\
& \hat{C}^{\prime}:=\sqrt{2}\left(I_{m}-\hat{D} \hat{D}^{\mathrm{T}}\right)^{-1 / 2}\left(I_{m}+D\right)^{-1} C
\end{aligned}
$$

Remark 3.2. An interesting special case of Theorem 3.2 is the case $D=I_{m}$. Since $\hat{D}=0$ (see (3.12)), (3.31) or, equivalently, (3.34) becomes

$$
0=\left(A-\frac{1}{2} B C\right) \mathscr{Q}+\mathscr{2}\left(A-\frac{1}{2} B C\right)^{\mathrm{T}}+\frac{1}{2} \mathscr{Q} C^{\mathrm{T}} C \mathscr{Q}+\frac{1}{2} B B^{\mathrm{T}} .
$$

Finally, we draw connections between Theorem 3.2 and the well-known positive real lemma used to characterize positive realness in the state-space setting [6]. 
Lemma 3.3. Let

$$
G(s) \sim\left[\begin{array}{l|l}
A & B \\
\hline C & D
\end{array}\right]
$$

be an $m \times m$ transfer function with minimal realization $(A, B, C, D)$. Then $G(s)$ is positive real if and only if there exist matrices $Q \in \mathbb{R}^{n \times n}, L \in \mathbb{R}^{n \times p}$, and $W \in \mathbb{R}^{m \times p}$ with $Q$ positive-definite and such that

$$
\begin{aligned}
& 0=A Q+Q A^{\mathrm{T}}+L L^{\mathrm{T}}, \\
& 0=Q C^{\mathrm{T}}-B+L W^{\mathrm{T}}, \\
& 0=D+D^{\mathrm{T}}-W W^{\mathrm{T}} .
\end{aligned}
$$

This form of the positive real lemma is the dual of that given in $[1,20]$, and the derivation is similarly dual. See [2] for further details on the dual positive real lemma.

The key question of interest here is the relationship between $Q$ satisfying (3.40)-(3.42) and 2 given by (3.31). To answer this question, we invoke the assumption that $D+D^{\mathrm{T}}>0$ which, as noted earlier, is needed for the existence of $\mathscr{Q}$. Thus, once again, we restrict our attention to strongly positive real transfer functions. In this case, it follows from (3.42) that

$$
W W^{\mathrm{T}}=D+D^{\mathrm{T}} .
$$

Now, since $D+D^{\mathrm{T}}>0, W$ is nonsingular and thus (3.41) implies (for square $W$ )

$$
L=\left(B-Q C^{\mathrm{T}}\right) W^{-\mathrm{T}} .
$$

Using (3.44) it follows from (3.40) that

$$
0=A Q+Q A^{\mathrm{T}}+\left(B-Q C^{\mathrm{T}}\right) W^{-\mathrm{T}} W^{-1}\left(B^{\mathrm{T}}-C Q\right)
$$

or, since $\left(W W^{\mathrm{T}}\right)^{-1}=W^{-\mathrm{T}} W^{-1}$,

$$
0=A Q+Q A^{\mathrm{T}}+\left(B-Q C^{\mathrm{T}}\right)\left(D+D^{\mathrm{T}}\right)^{-1}\left(B-Q C^{\mathrm{T}}\right)^{\mathrm{T}} .
$$

Thus, we have shown that under the assumption that $D+D^{\mathrm{T}}>0$, conditions (3.40)-(3.42) are equivalent to one Riccati equation given by (3.46). A similar result for the dual case appears in [6].

The next result connects the two Riccati equations (3.31) and (3.46).

Proposition 3.3. Assume $D+D^{\mathrm{T}}>0$. Then the Riccati equation (3.46) is identical to the Riccati equation (3.31), or, equivalently, (3.34).

Proof. Using (3.46) it follows that

$$
\begin{aligned}
0= & {\left[A-B\left(D+D^{\mathrm{T}}\right)^{-1} C\right] Q+Q\left[A-B\left(D+D^{\mathrm{T}}\right)^{-1} C\right]^{\mathrm{T}} } \\
& +Q C^{\mathrm{T}}\left(D+D^{\mathrm{T}}\right)^{-1} C Q+B\left(D+D^{\mathrm{T}}\right)^{-1} B^{\mathrm{T}} .
\end{aligned}
$$

The result now follows from algebraic manipulation by noting that

$$
\left(D+D^{\mathrm{T}}\right)^{-1}=2\left(I_{m}+D\right)^{-\mathrm{T}}\left[I_{m}-\left(D-I_{m}\right)\left(D+I_{m}\right)^{-1}\left(D+I_{m}\right)^{-\mathrm{T}}\left(D-I_{m}\right)^{\mathrm{T}}\right]^{-1}\left(I_{m}+D\right)^{-1} .
$$

Remark 3.3. Note that in the case $D=I_{m}$, Proposition 3.3 can readily be seen by comparing (3.39) and (3.46). 


\section{Robust stability problem with positive real uncertainty}

In this section we state the robust stability problem with positive real uncertainty. Consider the uncertain system

$$
\dot{x}(t)=\left[A-B_{0} F(t)\left(I_{m}+D_{0} F(t)\right)^{-1} C_{0}\right] x(t)
$$

where the uncertainty $F(\cdot)$ is characterized as

$$
\begin{aligned}
F(\cdot) \in \mathscr{F}:=\left\{F(\cdot): F(t)+F^{\mathrm{T}}(t)\right. & \geq 0 \text { and the elements of } F(\cdot) \text { are } \\
& \text { Lebesgue measurable on }[0, \infty)\},
\end{aligned}
$$

when the inverse of $I_{m}+D_{0} F(t)$ exists. For convenience we shall say that the matrix function $\hat{A}(\cdot)$ is asymptotically stable if the zero solution to the system $\dot{x}(t)=\hat{A}(t) x(t)$ is asymptotically stable. It is useful to note that $(\Sigma)$ can be viewed as a strongly positive real system $\left(A, B_{0}, C_{0}, D_{0}\right)$ in a negative feedback configuration with the time-varying gain $F(t)$ (see Figure 1). That is,

$$
\begin{aligned}
& \dot{x}(t)=A x(t)+B_{0} u(t), \\
& y(t)=C_{0} x(t)+D_{0} u(t),
\end{aligned}
$$

with negative feedback

$$
u(t)=-F(t) y(t),
$$

where

$$
F(\cdot) \in \mathscr{F} .
$$

Note that it follows from Lemma 3.2 that $\left(I_{m}+D_{0} F(t)\right)^{-1}$ exists when the system $\left(A, B_{0}, C_{0}, D_{0}\right)$ is strongly positive real. Thus, the question of interest is the stability of the uncertain system $(\Sigma)$ with positive real uncertainty (4.4).

Next, we present the main result of this section which shows that the uncertain system $(\Sigma)$ is robustly stable for all positive real uncertainty of the form (4.4). For the statement of the next result define the uncertainty set

$$
\mathscr{U}:=\left\{\Delta A(\cdot): \Delta A(t)=-B_{0} F(t)\left(I_{m}+D_{0} F(t)\right)^{-1} C_{0}, F(\cdot) \in \mathscr{F}\right\},
$$

where $B_{0} \in \mathbb{R}^{n \times m_{0}}, C_{0} \in \mathbb{R}^{m_{0} \times n}$, and $D_{0} \in \mathbb{R}^{m_{0} \times m_{0}}$ are fixed matrices denoting the structure of the uncertainty and $F(t) \in \mathbb{R}^{m_{0} \times m_{0}}$ is an uncertain matrix (see Figure 2).

In order to state the main result of this section we need the following lemma.

Lemma 4.1. Let

$$
G(s) \sim\left[\begin{array}{c|c}
A & B \\
\hline C & D
\end{array}\right]
$$

Then the following statements are equivalent:

(i) $A, A-B(I+D)^{-1} C$ are asymptotically stable and $G(s)$ is strongly positive real;

(ii) $D+D^{\mathrm{T}}>0$ and there exists a positive definite matrix $P$ such that

$$
0>A^{\mathrm{T}} P+P A+\left(C-B^{\mathrm{T}} P\right)^{\mathrm{T}}\left(D+D^{\mathrm{T}}\right)^{-1}\left(C-B^{\mathrm{T}} P\right) \text {. }
$$

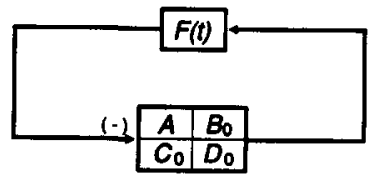

Fig. 1. 


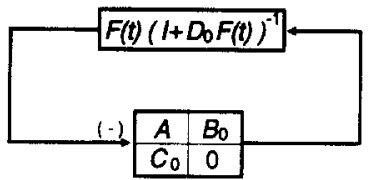

Fig. 2.

Proof. (i) $\Rightarrow$ (ii): Using the dual forms of Theorems 3.1 and 3.2 and Propositions 3.2 and 3.3, (i) is equivalent to

$$
I_{m}-\hat{B}^{\prime \mathrm{T}}\left(-s I_{n}-\hat{A}^{\prime}\right)^{-\mathrm{T}} \hat{C}^{\prime \mathrm{T}} \hat{C}^{\prime}\left(s I_{n}-\hat{A}^{\prime}\right)^{-1} \hat{B}^{\prime}>0 \text { for all } s=\mathrm{j} \omega .
$$

Next, let $\varepsilon>0$ be such that

$$
I_{m}-\hat{B}^{\prime \mathrm{T}}\left(-s I_{n}-\hat{A}^{\prime}\right)^{-\mathrm{T}}\left(\hat{C}^{\prime \mathrm{T}} \hat{C}^{\prime}+\varepsilon I_{n}\right)\left(s I_{n}-\hat{A}^{\prime}\right)^{-1} \hat{B}^{\prime}>0, \quad \text { for all } s=j \omega .
$$

Now, note that $\left(\hat{A}^{\prime}, \hat{C}^{\prime \mathrm{T}} \hat{C}^{\prime}+\varepsilon I_{n}\right)$ is observable and, since $\hat{A}-\frac{1}{2} \hat{B}^{\prime}\left(I-\hat{D}^{\mathrm{T}} \hat{D}\right)^{-1 / 2} \hat{D}^{\mathrm{T}} \hat{C}=A-B(I+D)^{-1} C$ and $A-B(I+D)^{-1} C$ is asymptotically stable, $\left(\hat{A}^{\prime}, \hat{B}^{\prime}\right)$ is stabilizable. Hence, it follows from Lemma 5 of [49] that there exists a real symmetric $P$ such that

$$
0=\hat{A}^{\prime \mathrm{T}} P+P \hat{A}^{\prime}+P \hat{B}^{\prime} \hat{B}^{\prime \mathrm{T}} P+\hat{C}^{\prime \mathrm{T}} \hat{C}^{\prime}+\varepsilon I_{n},
$$

or, equivalently, using the dual of Proposition 3.3,

$$
0=A^{\mathrm{T}} P+P A+\left(C-B^{\mathrm{T}} P\right)^{\mathrm{T}}\left(D+D^{\mathrm{T}}\right)^{-1}\left(C-B^{\mathrm{T}} P\right)+\varepsilon I_{n} .
$$

Now, since $A$ is assumed to be asymptotically stable and $\left(C-B^{\mathrm{T}} P\right)^{\mathrm{T}}\left(D+D^{\mathrm{T}}\right)^{-1}\left(C-B^{\mathrm{T}} P\right)+\varepsilon I_{n}>0$, it follows from Lyapunov theory that $P>0$ so that existence of a positive definite $P$ satisfying (4.6) is established.

(ii) $\Rightarrow$ (i): Suppose (ii) holds. Now note that

$$
G(s)+G^{*}(s)=\left[\begin{array}{cc|c}
-A^{\mathrm{T}} & 0 & -C^{\mathrm{T}} \\
0 & A & B \\
\hline B^{\mathrm{T}} & C & D+D^{\mathrm{T}}
\end{array}\right] .
$$

Next, if follows from (4.6) that there exists a positive definite $H$ such that

$$
-H=A^{\mathrm{T}} P+P A+\left(C-B^{\mathrm{T}} P\right)^{\mathrm{T}}\left(D+D^{\mathrm{T}}\right)^{-1}\left(C-B^{\mathrm{T}} P\right) .
$$

Applying the similarity transformation given by

$$
S=\left[\begin{array}{cc}
I_{n} & P \\
0 & I_{n}
\end{array}\right]
$$

to (4.11) we obtain

$$
G(s)+G^{*}(s) \sim\left[\begin{array}{cc|c}
-A^{\mathrm{T}} & A^{\mathrm{T}} P+P A & P B-C^{\mathrm{T}} \\
0 & A & B \\
\hline B^{\mathrm{T}} & -B^{\mathrm{T}} P+C & D+D^{\mathrm{T}}
\end{array}\right]
$$


or equivalently, using (4.12),

$$
\begin{aligned}
G(s)+G^{*}(s) & \sim\left[\begin{array}{cc|c}
-A^{\mathrm{T}} & -\left(C-B^{\mathrm{T}} P\right)^{\mathrm{T}}\left(D+D^{\mathrm{T}}\right)^{-1}\left(C-B^{\mathrm{T}} P\right)-H & P B-C^{\mathrm{T}} \\
0 & A & B \\
\hline B^{\mathrm{T}} & -B^{\mathrm{T} P}+C & D+D^{\mathrm{T}}
\end{array}\right] \\
& =N^{*}(s) N(s)+D+D^{\mathrm{T}}-\left(C-B^{\mathrm{T}} P\right) E^{-2}\left(C-B^{\mathrm{T} P}\right)^{\mathrm{T}},
\end{aligned}
$$

where

$$
E:=\left[\left(C-B^{\mathrm{T}} P\right)^{\mathrm{T}}\left(D+D^{\mathrm{T}}\right)^{-1}\left(C-B^{\mathrm{T}} P\right)+H\right]^{1 / 2}>0
$$

and

$$
N(s) \sim\left[\begin{array}{c|c}
A & B \\
\hline E & E^{-1}\left(C-B^{\mathrm{T} P}\right)^{\mathrm{T}}
\end{array}\right] .
$$

Noting that $D+D^{\mathrm{T}}-\left(C-B^{\mathrm{T}} P\right) E^{-2}\left(C-B^{\mathrm{T}} P\right)^{\mathrm{T}}>0$ it follows from $(4.14)$ that $G(\mathrm{j} \omega)+G^{*}(\mathrm{j} \omega)>0$, for all $\omega \in \mathbb{R}$. Next, note that since $P>0$ and $\left(C-B^{\mathrm{T}} P\right)^{\mathrm{T}}\left(D+D^{\mathrm{T}}\right)^{-1}\left(C-B^{\mathrm{T}} P\right)+H>0$ it follows from (4.12) that $A$ and $A-B(I+D)^{-1} C$ are asymptotically stable.

Theorem 4.1. Let

$$
G(s) \sim\left[\begin{array}{c|c}
A & B_{0} \\
\hline C_{0} & D_{0}
\end{array},\right.
$$

where $A \in \mathbb{R}^{n \times n}$ is asymptotically stable. If $G(s)$ is strongly positive real, then $A+\Delta A(\cdot)$ is asymptotically stable for all $\Delta A(\cdot) \in \mathscr{U}$.

Proof. It follows from Lemma 4.1 that if $G(s)$ is strongly positive real then there exist positive-definite $P$ and $R$ such that

$$
0=A^{\mathrm{T}} P+P A+\left(C_{0}-B_{0}^{\mathrm{T}} P\right)^{\mathrm{T}}\left(D_{0}+D_{0}^{\mathrm{T}}\right)^{-1}\left(C_{0}-B_{0}^{\mathrm{T}} P\right)+R,
$$

or, equivalently,

$$
\begin{aligned}
0= & {\left[A-B_{0} F(t)\left(I+D_{0} F(t)\right)^{-1} C_{0}\right]^{\mathrm{T}} P+P\left[A-B_{0} F(t)\left(I+D_{0} F(t)\right)^{-1} C_{0}\right] } \\
& +P B_{0} F(t)\left(I+D_{0} F(t)\right)^{-1} C_{0}+C_{0}^{\mathrm{T}}\left(I+D_{0} F(t)\right)^{-\mathrm{T}} F^{\mathrm{T}}(t) B_{0}^{\mathrm{T}} P \\
& +\left(C_{0}-B_{0}^{\mathrm{T}} P\right)^{\mathrm{T}}\left(D_{0}+D_{0}^{\mathrm{T}}\right)^{-1}\left(C_{0}-B_{0}^{\mathrm{T}} P\right)+R .
\end{aligned}
$$

We now show that system $(\Sigma)$ with $F(\cdot) \in \mathscr{F}$ is asymptotically stable with the Lyapunov function $V(x):=x^{\mathrm{T}} P x$. Indeed, the corresponding Lyapunov derivative is given by

$$
\dot{V}(x)=x^{\mathrm{T}}\left\{\left[A-B_{0} F(t)\left(I+D_{0} F(t)\right)^{-1} C_{0}\right]^{\mathrm{T}} P+P\left[A-B_{0} F(t)\left(I+D_{0} F(t)\right)^{-1} C_{0}\right]\right\} x
$$

or, equivalently, it follows from (4.16) that

$$
\dot{V}(x)=-x^{\mathrm{T}} \Omega x
$$


where

$$
\begin{aligned}
\Omega:= & P B_{0} F(t)\left(I+D_{0} F(t)\right)^{-1} C_{0}+C_{0}^{\mathrm{T}}\left(I+D_{0} F(t)\right)^{-\mathrm{T}} F^{\mathrm{T}}(t) B_{0}^{\mathrm{T}} P \\
& +\left(C_{0}-B_{0}^{\mathrm{T} P}\right)^{\mathrm{T}}\left(D_{0}+D_{0}^{\mathrm{T}}\right)^{-1}\left(C_{0}-B_{0}^{\mathrm{T}} P\right)+R .
\end{aligned}
$$

Next, add and subtract

$$
C_{0}^{\mathrm{T}}\left(I+D_{0} F(t)\right)^{-\mathrm{T}} F^{\mathrm{T}}(t)\left(D_{0}+D_{0}^{\mathrm{T}}\right)^{-1} F(t)\left(I+D_{0} F(t)\right)^{-1} C_{0}
$$

to and from $\Omega$ so that

$$
\begin{aligned}
\Omega= & P B_{0} F(t)\left(I+D_{0} F(t)\right)^{-1} C_{0}+C_{0}^{\mathrm{T}}\left(I+D_{0} F(t)\right)^{-\mathrm{T}} F^{\mathrm{T}}(t) B_{0}^{\mathrm{T}} P \\
& +\left(C_{0}-B_{0}^{\mathrm{T}} P\right)^{\mathrm{T}}\left(D_{0}+D_{0}^{\mathrm{T}}\right)^{-1}\left(C_{0}-B_{0}^{\mathrm{T}} P\right)+R \\
& +C_{0}^{\mathrm{T}}\left(I+D_{0} F(t)\right)^{-\mathrm{T}} F^{\mathrm{T}}(t)\left(D_{0}+D_{0}^{\mathrm{T}}\right)^{-1} F(t)\left(I+D_{0} F(t)\right)^{-1} C_{0} \\
& -C_{0}\left(I+D_{0} F(t)\right)^{-\mathrm{T}} F^{\mathrm{T}}(t)\left(D_{0}+D_{0}^{\mathrm{T}}\right)^{-1} F(t)\left(I+D_{0} F(t)\right)^{-1} C_{0} .
\end{aligned}
$$

Now, note that the last term in (4.21) can be rewritten (using some algebraic manipulations) as

$$
\begin{aligned}
& C_{0}^{\mathrm{T}}\left(I+D_{0} F(t)\right)^{-\mathrm{T}} F(t)\left[\left(I+D_{0} F(t)\right)^{-1}-I\right] C_{0} \\
& \quad+C_{0}^{\mathrm{T}}\left[\left(I-D_{0} F(t)\right)^{-\mathrm{T}}-I\right] F(t)\left(I+D_{0} F(t)\right)^{-1} C_{0}
\end{aligned}
$$

so that (4.21) becomes

$$
\begin{aligned}
\Omega= & \left(C_{0}-B_{0}^{\mathrm{T}} P\right)\left(D_{0}+D_{0}^{\mathrm{T}}\right)^{-1}\left(C_{0}-B_{0}^{\mathrm{T}} P\right)+R \\
& +P B_{0} F(t)\left(I+D_{0} F(t)\right)^{-1} C+C_{0}^{\mathrm{T}}\left(I+D_{0} F(t)\right)^{-\mathrm{T}} F^{\mathrm{T}}(t) B_{0}^{\mathrm{T}} P \\
& +C_{0}^{\mathrm{T}}\left(I+D_{0} F(t)\right)^{-\mathrm{T}} F^{\mathrm{T}}(t)\left(D_{0}+D_{0}^{\mathrm{T}}\right) F(t)\left(I+D_{0} F(t)\right)^{-1} C_{0} \\
& +C_{0}^{\mathrm{T}}\left(I+D_{0} F(t)\right)^{-1} F^{\mathrm{T}}(t)\left(I+D_{0} F(t)\right)^{-1} C_{0} \\
& \left.+C_{0}^{\mathrm{T}}\left(I+D_{0} F(t)\right)^{-1} C_{0}-C_{0}^{\mathrm{T}}\left(I+D_{0} F(t)\right)^{-\mathrm{T}} F^{\mathrm{T}}(t)\right) C_{0} .
\end{aligned}
$$

Next, grouping the appropriate terms in (4.23) yields

$$
\begin{aligned}
\Omega= & C_{0}^{\mathrm{T}}\left(I+D_{0} F(t)\right)^{-\mathrm{T}}\left(F(t)+F^{\mathrm{T}}(t)\right)\left(I+D_{0} F(t)\right)^{-1} C_{0} \\
& +C_{0}^{\mathrm{T}}\left(I+D_{0} F(t)\right)^{-\mathrm{T}} F^{\mathrm{T}}(t)\left(D_{0}+D_{0}^{\mathrm{T}}\right) F(t)\left(I+D_{0} F(t)\right)^{-1} C_{0}+R \\
& +\left(C_{0}-B_{0}^{\mathrm{T}} P\right)\left(D_{0}+D_{0}^{\mathrm{T}}\right)^{-1}\left(C_{0}-B_{0}^{\mathrm{T}} P\right) \\
& -\left(C_{0}^{\mathrm{T}}-P B_{0}\right) F(t)\left(I+D_{0} F(t)\right)^{-1} C_{0}-C_{0}^{\mathrm{T}}\left(I+D_{0} F(t)\right)^{-\mathrm{T}} F(t)\left(C_{0}-B_{0}^{\mathrm{T}} P\right)
\end{aligned}
$$

or, equivalently,

$$
\begin{aligned}
\Omega=R & +C_{0}^{\mathrm{T}}\left(I+D_{0} F(t)\right)^{-\mathrm{T}}\left(F(t)+F^{\mathrm{T}}(t)\right)\left(I+D_{0} F(t)\right)^{-1} C_{0} \\
+ & {\left[\left(D_{0}+D_{0}^{\mathrm{T}}\right)^{-1 / 2}\left(C_{0}-B_{0}^{\mathrm{T}} P\right)-\left(D_{0}+D_{0}^{\mathrm{T}}\right)^{1 / 2} F(t)\left(I+D_{0} F(t)\right)^{-1} C_{0}\right]^{\mathrm{T}} } \\
& \cdot\left[\left(D_{0}+D_{0}^{\mathrm{T}}\right)^{-1 / 2}\left(C_{0}-B_{0}^{\mathrm{T}} P\right)-\left(D_{0}+D_{0}^{\mathrm{T}}\right)^{1 / 2} F(t)\left(I+D_{0} F(t)\right)^{-1} C_{0}\right] .
\end{aligned}
$$

Thus it follows from (4.18) and (4.25) that $\dot{V}(x)=-x^{\mathrm{T}} \Omega x<0$ for all $F(\cdot) \in \mathscr{F}$, which shows that $A+\Delta A(\cdot)$ is asymptotically stable for all $\Delta A(\cdot) \in \mathscr{U}$. 
The key feature of the uncertainty set $\mathscr{U}$ is that the uncertain perturbation $\Delta A(\cdot)$ involves a phase constraint. To see this note that if $D_{0}+D_{0}^{\mathrm{T}}>0$ and $F(\cdot) \in \mathscr{F}$, then

$$
\begin{aligned}
& F(t)\left(I_{m}+D_{0} F(t)\right)^{-1}+\left[F(t)\left(I_{m}+D_{0} F(t)\right)^{-1}\right]^{\mathrm{T}} \\
& \quad=\left(I+D_{0} F(t)\right)^{-\mathrm{T}}\left[F(t)+F(t)^{\mathrm{T}}+F(t)^{\mathrm{T}}\left(D_{0}+D_{0}^{\mathrm{T}}\right) F(t)\right]\left(I+D_{0} F(t)\right)^{-1} \geq 0 .
\end{aligned}
$$

However, the term $F(t)\left(I_{m}+D_{0} F(t)\right)^{-1}$ is bounded in magnitude even though $F(t)$ is not. For example, if $F(t)$ is a scalar, then $\left|F(t)\left(1+D_{0} F(t)\right)^{-1}\right|<1 / D_{0}$. Thus the uncertainty set $\mathscr{U}$ incorporates both magnitude and phase constraints.

Next, we provide an alternative characterization of the uncertainty set $\mathscr{U}$. For this result, we assume for convenience that the uncertainty $F(\cdot) \in \mathscr{F}$ is time-invariant. In order to state this result we require some additional notation. Let

$$
\overline{\mathscr{F}}:=\left\{F \in \mathbf{R}^{m_{0} \times m_{0}}: F+F^{\mathrm{T}} \geq 0\right\}
$$

and

$$
\hat{\mathscr{F}}:=\left\{\hat{F}: \hat{F}=F\left(I+D_{0} F\right)^{-1}, F \in \bar{F}\right\}
$$

where $D_{0}+D_{0}^{\mathrm{T}}>0$ and by Lemma $3.2, \operatorname{det}\left(I+D_{0} F\right) \neq 0$. Note that $\hat{\mathscr{F}} \subset \overline{\mathscr{F}}$.

Proposition 4.1. Let $D_{0} \in \mathbb{R}^{m_{0} \times m_{0}}$ and assume $D_{0}+D_{0}^{\mathrm{T}}$ is positive definite. Then

$$
\hat{\mathscr{F}}=\left\{\hat{F} \in \mathbb{R}^{m_{0} \times m_{0}}: \operatorname{det}\left(I-\hat{F} D_{0}\right) \neq 0 \text { and } \hat{F}\left(D_{0}+D_{0}^{\mathrm{T}}\right) \hat{F}^{\mathrm{T}} \leq \hat{F}+\hat{F}^{\mathrm{T}}\right\} \text {. }
$$

Proof. ' $\subset$ ' Suppose $\hat{F} \in \hat{\mathscr{F}}$. Then there exists $F \in \bar{F}$ such that $\hat{F}=F\left(I+D_{0} F\right)^{-1}$. Hence $\hat{F} D_{0}=$ $F\left(I+D_{0} F\right)^{-1} D_{0}$ so that

$$
\begin{aligned}
\operatorname{spec}\left(\hat{F} D_{0}\right) & =\operatorname{spec}\left[F\left(I+D_{0} F\right)^{-1} D_{0}\right] \\
& =\operatorname{spec}\left[D_{0} F\left(I+D_{0} F\right)^{-1}\right]=\left\{\frac{\lambda}{1+\lambda}: \lambda \in \operatorname{spec}\left(D_{0} F\right)\right\},
\end{aligned}
$$

where 'spec' denotes spectrum. Hence, $1 \notin \operatorname{spec}\left(\hat{F} D_{0}\right)$ so that $\operatorname{det}\left(I-\hat{F} D_{0}\right) \neq 0$. Next note that $F=$ $\left(I-\hat{F} D_{0}\right)^{-1} \hat{F}$. Hence it follows that

$$
\begin{aligned}
\hat{F}+\hat{F}^{\mathrm{T}}-\hat{F}\left(D_{0}+D_{0}^{\mathrm{T}}\right) \hat{F}^{\mathrm{T}} & =\hat{F}+\hat{F}^{\mathrm{T}}-\hat{F} D_{0} \hat{F}^{\mathrm{T}}-\hat{F} D_{0}^{\mathrm{T}} \hat{F}^{\mathrm{T}} \\
& =\hat{F}\left(I-\hat{F} D_{0}\right)^{\mathrm{T}}+\left(I-\hat{F} D_{0}\right) \hat{F}^{\mathrm{T}} \\
& =\left(I-\hat{F} D_{0}\right)\left[\left(I-\hat{F} D_{0}\right)^{-1} \hat{F}+\hat{F}^{\mathrm{T}}\left(I-\hat{F} D_{0}\right)^{-\mathrm{T}}\right]\left(I-\hat{F} D_{0}\right)^{\mathrm{T}} \\
& =\left(I-\hat{F} D_{0}\right)\left(F+F^{\mathrm{T}}\right)\left(I-\hat{F} D_{0}\right)^{\mathrm{T}} \\
& \geq 0 .
\end{aligned}
$$

This proves ' $\subset$ '. 
' $\supset$ ' Let $\hat{F}$ be such that $\operatorname{det}\left(I-\hat{F} D_{0}\right) \neq 0$ and $\hat{F}\left(D_{0}+D_{0}^{\mathrm{T}}\right) \hat{F}^{\mathrm{T}} \leq \hat{F}+\hat{F}^{\mathrm{T}}$. Since $\operatorname{det}\left(I-\hat{F} D_{0}\right) \neq 0$, define $F:=\left(I-\hat{F} D_{0}\right)^{-1} \hat{F}$. Then it follows that

$$
\begin{aligned}
F+F^{\mathrm{T}} & =\left(I-\hat{F} D_{0}\right)^{-1} \hat{F}+\hat{F}^{\mathrm{T}}\left(I-\hat{F} D_{0}\right)^{-\mathrm{T}} \\
& =\left(I-\hat{F} D_{0}\right)^{-1}\left[\hat{F}\left(I-\hat{F} D_{0}\right)^{\mathrm{T}}+\left(I-\hat{F} D_{0}\right) \hat{F} \mathrm{~T}\right]\left(I-\hat{F} D_{0}\right)^{\mathrm{T}} \\
& =\left(I-\hat{F} D_{0}\right)^{-1}\left[\hat{F}+\hat{F}^{\mathrm{T}}-\hat{F}\left(D_{0}+D_{0}^{\mathrm{T}}\right) \hat{F}^{\mathrm{T}}\right]\left(I-\hat{F} D_{0}\right)^{-\mathrm{T}} \\
& \geq 0 .
\end{aligned}
$$

Hence $F \in \overline{\mathscr{F}}$. Furthermore since $F=\left(I-\hat{F} D_{0}\right)^{-1} \hat{F}$ is equivalent to $\hat{F}=F\left(I+D_{0} F\right)^{-1}, \hat{F} \in \hat{\mathscr{F}}$.

Remark 4.1. It is shown in Section 5 that a natural characterization of uncertainty that can be captured by (4.5) arises in lightly damped structures with uncertain modal data. An uncertainty structure similar to (4.28) was considered in [41] for modeling uncertainties as sector-bounded nonlinearities. However, no positive real uncertainty interpretation was provided in [41].

\section{Sufficient conditions for robust controller synthesis with positive real uncertainty}

In this section we state the Robust Stabilizability Problem With Positive Real Uncertainty. The problem involves the set $\mathscr{U}$ given by (4.5) of uncertain perturbations $\Delta A(\cdot)$ of the nominal $(A, B, C)$ system. The goal of the robust stability problem is to determine a static output feedback controller that stabilizes the plant for all variations in $\mathscr{U}$. See Figure 3.

Robust Stabilizability Problem With Positive Real Uncertainty. Determine $K \in \mathbb{R}^{m \times I}$ such that the closed-loop system consisting of the $n$ th-order controlled plant

$$
\dot{x}(t)=(A+\Delta A(t)) x(t)+B u(t), \quad t \in[0, \infty),
$$

measurements

$$
y(t)=C x(t),
$$

and output feedback controller

$$
u(t)=K y(t),
$$

is asymptotically stable for all $\Delta A(\cdot) \in \mathscr{U}$.

For each uncertain variation $\Delta A(\cdot) \in \mathscr{U}$, the closed-loop system can be written as

$$
\dot{x}(t)=(A+B K C+\Delta A(t)) x(t), \quad t \in[0, \infty) .
$$

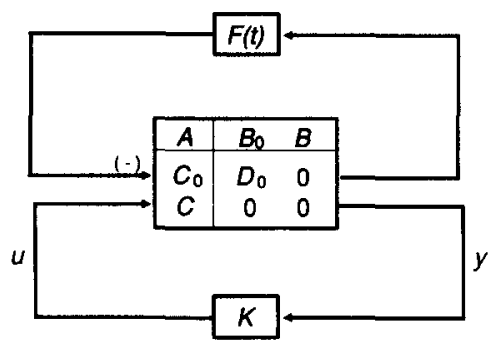

Fig. 3. 
The following result gives sufficient conditions for constructing a feedback gain $K$ that solves the Robust Stabilizability Problem With Positive Real Uncertainty. For the statement of this result define

$$
\nu:=Q C^{\mathrm{T}}\left(C Q C^{\mathrm{T}}\right)^{-1} C, \quad \nu_{\perp}:=I_{n}-\nu, \quad R_{0}:=\left(D_{0}+D_{0}^{\mathrm{T}}\right)^{-1},
$$

for arbitrary $Q \in \mathbb{R}^{n \times n}$ such that det $C Q C^{\mathrm{T}} \neq 0$, and let $R, R_{1}$ and $R_{2}$ be arbitrary real $n \times n$ and $m \times m$ positive-definite matrices.

Theorem 5.1. Suppose there exist $n \times n$ nonnegative-definite matrices $Q, P$ satisfying

$$
\begin{aligned}
0= & \left(A-B R_{2}^{-1} B^{\mathrm{T}} P \nu-B_{0} R_{0} C_{0}\right) Q+Q\left(A-B R_{2}^{-1} B^{\mathrm{T}} P \nu-B_{0} R_{0} C_{0}\right)^{\mathrm{T}} \\
& +Q C_{0}^{\mathrm{T}} R_{0} C_{0} Q+B_{0} R_{0} B_{0}^{\mathrm{T}}+R, \\
0= & \left(A-B_{0} R_{0} C_{0}+Q C_{0}^{\mathrm{T}} R_{0} C_{0}\right)^{\mathrm{T}} P+P\left(A-B_{0} R_{0} C_{0}+Q C_{0}^{\mathrm{T}} R_{0} C_{0}\right) \\
& +R_{1}-P B R_{2}^{-1} B \mathrm{~T} P+\nu_{\perp}^{\mathrm{T}} P B R_{2}^{-1} B^{\mathrm{T}} P \nu_{\perp},
\end{aligned}
$$

and let $K$ be given by

$$
K=-R_{2}^{-1} B^{\mathrm{T}} P Q C^{\mathrm{T}}\left(C Q C^{\mathrm{T}}\right)^{-1} .
$$

Then $A+B K C+\Delta A(\cdot)$ is asymptotically stable for all $\Delta A(\cdot) \in \mathscr{U}$.

Proof. With $K$ given by (5.7), it follows that (5.5) is equivalent to

$$
\begin{aligned}
0= & \left(A+B K C-B_{0} R_{0} C_{0}\right) Q+Q\left(A+B K C-B_{0} R_{0} C_{0}\right)^{\mathrm{T}} \\
& +Q C_{0}^{\mathrm{T}} R_{0} C_{0} Q+B_{0} R_{0} B_{0}^{\mathrm{T}}+R,
\end{aligned}
$$

which further implies

$$
\begin{aligned}
0= & (A+B K C) Q+Q(A+B K C)^{\mathrm{T}} \\
& +\left(B_{0}-Q C_{0}^{\mathrm{T}}\right)\left(D_{0}+D_{0}^{\mathrm{T}}\right)^{-1}\left(B_{0}-Q C_{0}^{\mathrm{T}}\right)^{\mathrm{T}}+R .
\end{aligned}
$$

Furthermore, (5.6) is equivalent to

$$
\begin{aligned}
0= & \left(A+B K C-B_{0} R_{0} C_{0}+Q C_{0}^{\mathrm{T}} R_{0} C_{0}\right)^{\mathrm{T}} P \\
& +P\left(A+B K C-B_{0} R_{0} C_{0}+Q C_{0}^{\mathrm{T}} R_{0} C_{0}\right)+R_{1}+K^{\mathrm{T}} R_{2} K .
\end{aligned}
$$

Note that (5.10) is an auxiliary equation and is only needed for computing the gain $K$. Furthermore, note that (5.9) is equivalent to (3.46), or, equivalently (3.31). It now follows from Lemma 4.1 that $(A+$ $\left.B K C, B_{0}, C_{0}, D_{0}\right)$ is strongly positive real which, by Theorem 4.1 , implies that $A+B K C+\triangle A(\cdot)$ is asymptotically stable for all $\Delta A(\cdot) \in \mathscr{U}$.

Remark 5.1. Theorem 5.1 presents sufficient conditions for designing robust controllers for a time-varying positive real uncertainty structure. These conditions are derived by first obtaining necessary conditions for an auxiliary optimization problem in the spirit of $[14,23]$ and then showing by construction that these conditions serve as sufficient conditions for robust stabilization with positive real uncertainty. Specifically, we minimize $\mathscr{J}(K):=\operatorname{tr} Q\left(R_{1}+C^{\mathrm{T}} K^{\mathrm{T}} R_{2} K C\right)$ subject to $Q$ satisfying (5.9). It can be shown that $\mathscr{J}(K)$ is an upper bound to an $\mathrm{H}_{2}$ cost functional thus providing a worst-case performance bound.

Next, we specialize Theorem 5.1 to the full-state feedback case. When the full state is available, i.e., $C=I_{n}$, the projection $\nu=I_{n}$ so that $\nu_{\perp}=0$. In this case (5.7) becomes

$$
K=-R_{2}^{-1} B^{\mathrm{T}} P
$$


and (5.5), (5.6) specialize to

$$
\begin{aligned}
0= & \left(A-B R_{2}^{-1} B^{\mathrm{T}} P-B_{0} R_{0} C_{0}\right) Q \\
& +Q\left(A-B R_{2}^{-1} B^{\mathrm{T}} P-B_{0} R_{0} C_{0}\right)^{\mathrm{T}}+Q C_{0}^{\mathrm{T}} R_{0} C_{0} Q+B_{0} R_{0} B_{0}^{\mathrm{T}}+R, \\
0= & \left(A-B_{0} R_{0} C_{0}+Q C_{0}^{\mathrm{T}} R_{0} C_{0}\right)^{\mathrm{T}} P \\
& +P\left(A-B_{0} R_{0} C_{0}+Q C_{0}^{\mathrm{T}} R_{0} C_{0}\right)+R_{1}-P B R_{2}^{-1} B^{\mathrm{T}} P .
\end{aligned}
$$

Note that even in the full-state feedback case the result involves two coupled Riccati equations. This should not be surprising since as pointed out in Remark 5.1 the results were obtained via an auxiliary optimization problem. Similarly, the $\mathrm{H}_{2} / \mathrm{H}_{\infty}$ full-state feedback problem considered in [23] also involved two coupled equations.

A salient feature of (3.39) is the fact that the shift $-\frac{1}{2} B C$ to the matrix $A$ can be nonpositive. That is, $-\frac{1}{2} B C$ can represent a left shift in contrast to the usual $\alpha$-shift, which is a uniform open-loop right shift used to place the closed-loop poles to the left of $-\alpha$, where $\alpha>0$ [7]. The use of a left shift to the plant dynamics matrix has been used to model frequency uncertainty in lightly damped flexible structures [11-13,27,28]. Specifically, consider modal dynamics of the form

$$
A=\text { block-diag }\left(\left[\begin{array}{rr}
-\eta_{1} & \omega_{1} \\
-\omega_{1} & -\eta_{1}
\end{array}\right], \ldots,\left[\begin{array}{rr}
-\eta_{r} & \omega_{r} \\
-\omega_{r} & -\eta_{r}
\end{array}\right]\right)
$$

where $\eta_{i}>0$ denotes the decay rate and $\omega_{i}$ denotes modal frequency. Also consider uncertainty of the form

$$
\Delta A=\sum_{i=1}^{r} \sigma_{i} A_{i},
$$

where $\sigma_{i} \in\left[-\delta_{i}, \delta_{i}\right], i=1, \ldots, r$, are real, uncertain parameters with given bounds $\delta_{i}$, and the matrices $A_{i}$ are defined by

$$
A_{i}=\text { block-diag }\left(0, \ldots, 0,\left[\begin{array}{rr}
0 & 1 \\
-1 & 0
\end{array}\right], 0, \ldots, 0\right),
$$

where the matrix $\left[\begin{array}{rr}0 & 1 \\ -1 & 0\end{array}\right]$ corresponds to the $i$-th diagonal block of $A$. The skew symmetric structure of $A_{i}$ accounts for uncertainty in the $i$-th modal frequency $\omega_{i}$. In [11-13,16,18,27,28] the Maximum Entropy design approach is predicated upon a modified covariance (Lyapunov) equation of the form

$$
0=(A+S) Q+Q(A+S)^{\mathrm{T}}+\sum_{i=1}^{r} \delta_{i}^{2} A_{i} Q A_{i}^{\mathrm{T}}+V,
$$

where the shift $S$ is defined by $S:=\frac{1}{2} \sum_{i=1}^{r} \delta_{i}^{2} A_{i}^{2}$. Note that $S$ has the form

$$
S=\text { block-diag }\left(-\frac{1}{2} \delta_{1}^{2} I_{2}, \ldots,-\frac{1}{2} \delta_{r}^{2} I_{2}\right)
$$

so that $S$ effectively shifts each mode to the left by introducing a (fictitious) augmentation to the open-loop damping. To relate (5.18) to (3.39), consider the case of a single uncertain modal frequency by setting $r=1$. Furthermore, let

$$
B_{0}=C_{0}=\delta_{1} I_{2}, \quad A_{1}=\left[\begin{array}{rr}
0 & 1 \\
-1 & 0
\end{array}\right],
$$

so that (with $B, C$ replaced by $B_{0}, C_{0}$ in (3.39)) $-\frac{1}{2} B_{0} C_{0}=-\frac{1}{2} \delta_{1}^{2} I_{2}=\frac{1}{2} \delta_{1}^{2} A_{1}^{2}=S$. The remaining terms $\delta_{1}^{2} A_{1} Q A_{1}^{\mathrm{T}}+V$ in (5.18) can be shown to play a role similar to the terms $\frac{1}{2} \mathscr{Q} C^{\mathrm{T}} C \mathscr{Q}+\frac{1}{2} B B^{\mathrm{T}}$ in (3.39). See [15] for further details. Finally, the uncertain perturbations $\Delta A$ given by (4.5) (for constant $F$ ) have the form

$$
\Delta A=-\delta_{1}^{2} F\left(I_{2}+D_{0} F\right)^{-1} \text {. }
$$


In the limiting case $D_{0} \rightarrow 0$, setting $F=-\left(\sigma_{1} / \delta_{1}^{2}\right) A_{1}$ (so that $\left.F+F^{\mathrm{T}} \geq 0\right)$, (5.19) becomes

$$
\Delta A=\sigma_{1} A_{1} .
$$

Hence $\mathscr{U}$ given by (4.5) (for constant $F$ ) can be used to capture frequency uncertainty of the form (5.16).

\section{Acknowledgement}

The authors would like to thank the reviewer for providing several helpful suggestions and for bringing reference [41] to their attention.

\section{References}

[1] B.D.O. Anderson, A system theory criterion for positive real matrices, SIAM J. Control Optim. 5 (1967) 171-182.

[2] B.D.O. Anderson, Dual form of a positive real lemma, Proc. IEEE 55 (1967) 1749-1750.

[3] B.D.O. Anderson and J.B. Moore, Algebraic structure of generalized positive real matrices, SIAM J. Control 6 (1968) $615-624$.

[4] B.D.O. Anderson, A simplified viewpoint of hyperstability, IEEE Trans. Autom. Control 13 (1968) $292-294$.

[5] B.D.O. Anderson, The small gain theorem, the passivity theorem, and their equivalence, J. Franklin Inst. 293 (1972) $105-115$.

[6] B.D.O. Anderson and S. Vongpanitlerd, Network Analysis and Synthesis: A Modern Systems Theory Approach (Prentice-Hall, Englewood Cliffs, NJ, 1973).

[7] B.D.O. Anderson and J.B. Moore, Optimal Control Linear Quadratic Methods (Prentice-Hall, Englewood Cliffs, NJ, 1990).

[8] M.J. Balas, Direct velocity feedback control of large space structures, J. Guidance Control 2 (1967) 252-253.

[9] J.R. Bar-On and E.A. Jonckheere, Phase margins for multivariable control systems, Internat. J. Control 52 (1990) $485-498$.

[10] R.J. Benhabib, R.P. Iwens and R.L. Jackson, Stability of large space structure control systems using positivity concepts, $J$. Guidance Control 4 (1981) 487-494.

[11] D.S. Bernstein and S.W. Greeley, Robust controller synthesis using the maximum entropy design equations, IEEE Trans. Automat. Control 31 (1986) 362-364.

[12] D.S. Bernstein and D.C. Hyland, The optimal projection/maximum entropy approach to designing low-order, robust controllers for flexible structures, Proc. Conf. Decision and Control Fort Lauderdale, FL (1985) 745-752.

a. D.S. Bernstein and D.C. Hyland, The optimal projection approach to robust, fixed-structure control design, in: J.L. Junkins, Ed., Mechanics and Control of Space Structures (AIAA 1990) 379-380.

[13] D.S. Bernstein and D.C. Hyland, Optimal projection for uncertain systems (OPUS): A unified theory of reduced-order, robust control design, in: S.N. Atluri and A.K. Amos, Eds., Large Space Structures: Dynamics and Control (Springer-Verlag, Berlin-New York, 1988) 263-302.

[14] D.S. Bernstein and W.M. Haddad, LQG control with an $H_{\infty}$ performance bound: a Riccati equation approach, IEEE Trans. Automat. Control 34 (1989) 293-305.

[15] D.S. Bernstein and W.M. Haddad, Robust stability and performance analysis for state space systems via quadratic Lyapunov bounds, SIAM J. Matrix Anal. Appl. 11 (1990) 239-271.

[16] D.S. Bernstein, E.G. Collins, Jr. and D.C. Hyland, Real parameter uncertainty and phase information in the robust control of flexible structures, Proc. Conf. Decision Control, Honolulu, HI (1990) 379-380.

[17] S. Boyd and Q. Yang, Structured and simultaneous Lyapunov functions for system stability problems, Internat. J. Control 49 (1989) 2215-2240.

[18] M. Cheung and S. Yurkovich, On the robustness of MEOP design versus asymptotic LQG synthesis, IEEE Trans. Automat. Control 33 (1988) 1061-1065.

[19] C.A. Desoer and M. Vidyasagar, Feedback Systems: Input-Output Properties (Academic Press, New York, 1975).

[20] B.W. Dickinson, Analysis of the Lyapunov equation using generalized positive real matrices, IEEE Trans. Automat. Control 25 (1980) 560-563.

[21] J.C. Doyle, K. Glover, P.P. Khargonekar and B.A. Francis, State-space solutions to standard $\mathrm{H}_{2}$ and $\mathrm{H}_{\infty}$ control problems, IEEE Trans. Automat. Control 34 (1989) 831-847.

[22] B.A. Francis, $A$ Course in $H_{\infty}$ Control Theory (Springer-Verlag, Berlin-New York, 1987).

[23] W.M. Haddad and D.S. Bernstein, On the gap between $\mathrm{H}_{2}$ and entropy performance measures in $\mathrm{H}_{\infty}$ control, Systems Control Lett. 14 (1990) 113-120.

[24] W.M. Haddad and D.S. Bernstein, Generalized Riccati equations for the full- and reduced-order mixed-norm $\mathrm{H}_{2} / \mathrm{H}_{\infty}$ standard problem, Systems Control Lett. 14 (1990) 185-197.

[25] G. Hewer and C. Kenney, Dissipative LQG control systems, IEEE Trans. Automat. Control 34 (1989) $866-870$.

[26] D.J. Hill and P.J. Moylan, Dissipative dynamical systems: Basic input-output and state properties, J. Franklin Inst. 309 (1980) 327-357. 
[27] D.C. Hyland, Minimum entropy stochastic modelling of linear systems with a class of parameter uncertainties, Proc. Amer. Control Conf., Arlington, VA (1982) 620-627.

[28] D.C. Hyland, Maximum entropy stochastic approach to controller design for uncertain structural systems, Proc. Amer. Control Conf., Arlington, VA (1982) 680-688.

[29] S.M. Joshi, Robustness properties of collocated controllers for flexible spacecraft, J. Guidance Control 9 (1986) $85-91$.

[30] S.M. Joshi, Control of Large Flexible Space Structures (Springer-Verlag, Berlin-New York, 1989).

[31] M.J. Jacobus, Stable, fixed-order dynamic compensation with applications to positive real and $\mathrm{H}^{\infty}$-constrained control design, Ph.D. Dissertation, Univ. of New Mexico (1990).

[32] P.P. Khargonekar, I.R. Petersen and K. Zhou, Robust stabilization of uncertain linear systems: Quadratic stabilizability and H $^{\infty}$ control theory, IEEE Trans. Automat. Control 35 (1990) 356-361.

[33] Y.D. Landau, Adaptive Control: The Model Reference Approach (Dekker, New York, 1979).

[34] R.L. Leal and S.M. Joshi, On the design of dissipative LQG-type controllers, Proc. Conf. Decision and Control Austin, TX (1988) $1645-1646$.

[35] L. Lee and A.L. Tits, Robustness under uncertainty with phase information, Proc. Conf. Decision and Control Tampa, FL (1989) 2315-2316.

[36] R. Lozano-Leal and S. Joshi, Strictly positive real transfer functions revisited, IEEE Trans. Automat. Control 35 (1990) 1243-1245.

[37] D.G. MacMartin and S.R. Hall, An $\mathrm{H}_{\infty}$ power flow approach to the control of uncertain structures, Proc. Amer. Control Conf., San Diego, CA (1990) 3073-3080.

[38] M.D. McLaren and G.L. Slater, Robust multivariable control of large space structures using positivity, J. Guidance Control Dyn. 10 (1987) 393-400.

[39] K.S. Narendra and H.J. Taylor, Frequency Domain Criteria for Absolute Stability (Academic Press, New York, 1973).

[40] R.W. Newcomb, Linear Multiport Synthesis (McGraw-Hill, New York, 1966).

[41] E. Noldus, Design of robust state feedback laws, Internat. J. Control 35 (1982) 935-944.

[42] I.R. Petersen, Disturbance attenuation and $\mathrm{H}^{\infty}$ optimization: A design method based on the algebraic Riccati equation, IEEE Trans. Automat. Control 32 (1987) 427-429.

[43] V.M. Popov, Hyperstability of Automatic Control Systems (Springer-Verlag, Berlin-New York, 1973).

[44] I. Postlethwaite, J.M. Edmunds and A.G.J. MacFarlane, Principal gains and principal phases in the analysis of linear multivariable feedback systems, IEEE Trans. Automat. Control 26 (1981) 32-46.

[45] M.G. Safonov, E.A. Jonckheere and D.J.N. Limebeer, Synthesis of positive real multivariable feedback systems, Internat. J. Control 45 (1987) 817-842.

[46] D.D. Siljak, New algebraic criteria for positive realness, J. Franklin Inst. 291 (1971) 109-120.

[47] G. Tao and P.A. Ioannou, Strictly positive real matrices and the Lefschetz-Kalman-Yakubovich lemma, IEEE Trans. Automat Control 33 (1988) 1183-1185.

[48] J.T. Wen, Time domain and frequency domain conditions for strict positive realness, IEEE Trans. Automat Control 33 (1988) 988-992.

[49] J.C. Willems, Least squares stationary optimal control and the algebraic Riccati equation, IEEE Trans. Automat. Control 16 (1971) 621-634.

[50] J.C. Willems, Dissipative dynamical systems part I: General theory, Arch. Rational Mech. 45 (1972) $321-351$.

[51] J.C. Willems, Dissipative dynamical systems part II: Quadratic supply rates, Arch. Rational Mech. 45 (1972) $352-393$.

[52] H.K. Wimmer, Monotonicity of maximal solutions of algebraic Riccati equations, Systems Control Lett. 5 (1985) $317-319$.

[53] G. Zames, On the input-output stability of time-varying nonlinear feedback systems part I: Condition derived using concepts of loop gain, conicity, and positivity, IEEE Trans. Automat. Control 11 (1966) 228-238.

[54] K. Zhou and P.P. Khargonekar, An algebraic Riccati equation approach to $\mathrm{H}^{\infty}$ optimization, Systems Control Lett. 11 (1988) $85-91$. 